\title{
Superelastic load cycling of Gum Metal
}

\author{
V.A. Vorontsov ${ }^{\mathrm{a}}$, N.G. Jones ${ }^{\mathrm{b}}$, K.M. Rahman ${ }^{\mathrm{a}}$, D. Dye ${ }^{\mathrm{a}}$ \\ ${ }^{a}$ Department of Materials, Royal School of Mines, Imperial College, Prince Consort Road, South \\ Kensington, London SW7 2BP, UK \\ ${ }^{\mathrm{b}}$ Department of Materials Science and Metallurgy, University of Cambridge, 27 Charles Babbage \\ Road, Cambridge, CB3 OFS, UK
}

\begin{abstract}
The superelastic beta titanium alloy, Gum Metal, has been found to accumulate plastic strain during tensile load cycling in the superelastic regime. This is evident from the positive drift of the macroscopic stress vs. strain hysteresis curve parallel to the strain axis and the change in its geometry subsequent to every load-unload cycle. In addition, there is a progressive reduction in the hysteresis loop width and in the stress at which the superelastic transition occurs. In-situ synchrotron X-ray diffraction has shown that the lattice strain exhibited the same behaviour as that observed in macroscopic measurements and identified further evidence of plastic strain accumulation. The mechanisms responsible for the observed behaviour have been evaluated using transmission electron microscopy, which revealed a range of different defects that formed during load cycling. The formation of these defects is consistent with the classical mathematical theory for the b.c.c. to orthorhombic martensitic transformation. It is the accumulation of these defects over time that alters its superelastic behaviour.
\end{abstract}

Key words: Gum metal; beta titanium alloys; martensitic transformation; synchrotron X-ray scattering; transmission electron microscopy

\section{Introduction}

In 2003, Saito et al. [1] presented their findings on a novel metastable $\beta$-titanium alloy (Ti-36Nb-2Ta-3Zr-0.3O wt.\%), Gum Metal, that exhibited a unique combination of attractive mechanical properties. These included a high tensile strength in excess of $1 \mathrm{GPa}$, a low elastic modulus of $\approx 70 \mathrm{GPa}$ in the hot worked condition $(\approx 55 \mathrm{GPa}$ after cold rolling) as well as superelastic and superplastic behaviour. These 'super' properties were attained by selecting a composition that simultaneously satisfied three theoretically predicted electronic parameters: electron per atom ratio $(e / a=4.24)$, bond order $(B o=2.87)$ and $d$ electron orbital energy level $(\mathrm{Md}=2.45 \mathrm{eV})$. 
The authors noted that the material exhibited very little hardening even after substantial cold work. Based on this and their microstructural observations, it was stipulated that the material underwent plastic deformation via a dislocation free mechanism. They claimed that no martensitic transformation took place during cold work. Instead, the unstable $\beta$ lattice readily formed giant faults by ideal shear and thus accommodated the plastic strain. However, the in-situ synchrotron X-ray work of Talling et al. [2,3], has conclusively proven that a reversible stress induced martensitic transformation, whereby the body-centred cubic (bcc) $\beta$ phase transforms to the orthorhombic $\alpha^{\prime \prime}$, is responsible for the superelastic behaviour.

Despite this, the mechanisms responsible for the peculiar mechanical behaviour of Gum metal are complex and remain a subject of ongoing work. Morris et al. [4] have shown that the propensity of Gum Metal for undergoing the martensitic transformation is orientation and texture dependent. Furthermore, structures commonly referred to as "giant faults", nanodisturbances, twinning, dislocations and $\alpha^{\prime \prime}$ are typically observed in the $\beta$ phase after cold working of Gum Metal as well as other superelastic alloys like Ti-2448 and Ti-12Mo. Their exact role in the deformation processes remains to be determined, while there has been no evidence to date that their origin is assisted by dislocation glide $[1,5]$. Furthermore, cold work results in the formation of large number of fine stress-induced $\omega$ phase precipitates $[6-8]$. These have a hexagonal crystal structure that results from the systematic collapse of the $\{111\}$ planes in the $\beta$ phase. Since cold working increases the tensile strength of Gum Metal, it is likely that the formation of the $\omega$ phase plays an important role. Lastly, plasticity that is mediated by dislocation glide has also been reported in derivative alloys $[9,10,5]$.

Due to its low elastic modulus and the low toxicity of niobium as a $\beta$ stabilising element [11], Gum Metal is receiving significant attention from the biomedical community as a candidate material for orthopaedic implants. In addition, the low modulus and the possibility of hysteretic superelastic behaviour make the alloy interesting to engineers for energy absorbing applications. Therefore, it is important to understand fully the mechanical behaviour of Gum Metal during superelastic load cycling.

In this work we examine the superelastic behaviour of Gum Metal under cyclic tensile loading using synchrotron X-ray diffraction and high-resolution electron microscopy. We show that the $\beta \rightarrow \alpha^{\prime \prime}$ martensitic transformation introduces a variety of permanent lattice defects. This leads to accumulation of plastic strain and changes the shape of superelastic hysteresis with every load-unload cycle. Finally, we provide an explanation for this accumulation of defects by employing classical mathematical theory to evaluate the martensitic transformation in Gum Metal.

\section{Experimental method}

\subsection{Material preparation}

The Gum Metal employed in this study was produced using ingot metallurgy. An elemental powder compact was melted in a high purity argon atmosphere using a helium plasma torch. The initial ingot was then triple remelted with inversions. A billet $60 \mathrm{~mm}$ in diameter was machined from the button-shaped ingot. It was then subjected to a 60 minute solution heat treatment at $850^{\circ} \mathrm{C}$. Deltaglaze ${ }^{\mathrm{TM}} 3418$ (Acheson, MI, USA) glass 
lubricant, was subsequently applied to the surface of the billet and it was extruded into $12 \mathrm{~mm}$ rod after a 105 minute heat treatment at $975^{\circ} \mathrm{C}$. Inductively coupled plasma optical emission spectrometry (ICP-OES) was used to verify the chemical composition of the final product: Ti-36.2Nb-1.96Ta-3.16Zr-0.26O wt.\%. (LECO analysers were used to measure the oxygen and hydrogen content.)

\subsection{In-situ synchrotron X-ray scattering}

The in-situ loading experiment was performed at the I12 beamline of the Diamond Light Source synchrotron X-ray facility in Didcot, Oxfordshire, UK. Figure 1 shows a schematic representation of the experimental set-up. A 'dog bone' tensile test specimen, with gauge dimensions of $1.5 \times 1.5 \times 19 \mathrm{~mm}$ was electric discharge machined (EDM) from the extruded Gum Metal bar, with the tensile axis aligned parallel to the extrusion direction. It was subjected to sub-yield cyclic tensile loading between 15 and $700 \mathrm{MPa}$ on a purpose built $5 \mathrm{kN}$ frame using a loading rate of $4 \mathrm{MPas}^{-1}$. The maximum stress does not exceed the yield strength of Gum Metal as can be seen from the full stress vs. strain curve in Figure 2. A total of 20 load-unload cycles were carried out. The macroscopic strain was recorded using a $10 \mathrm{~mm}$ contact extensometer.

The Debeye-Scherrer diffraction rings formed by the monochromated incident X-ray beam $(0.5 \times 0.5 \mathrm{~mm}, 80 \mathrm{keV}(\lambda=0.15498 \AA))$ were imaged using a Thales Pixium RF4343 $2880 \times 2880$ pixel $2 \mathrm{D}$ area detector positioned $1320.725 \mathrm{~mm}$ from the specimen. The images were acquired using $2 \mathrm{~s}$ exposures, while the detector's write time was a further $0.5 \mathrm{~s}$ per image.

The diffraction ring images were processed using the FIT2D [12] analysis software to obtain the intensity vs. $2 \theta$ (scattering angle) spectra. The data are taken from a $10^{\circ}$ azimuthal bin around $90^{\circ}$, i.e. aligned to the tensile axis. The instrument parameters necessary for the analysis were determined using a powder standard. The Wavemetrics IGOR Pro software package was then used to perform fitting of a Gaussian function to the individual lattice peaks observed in the integrated spectra.

\subsection{Post-mortem electron microscopy}

Screw-threaded fatigue specimens with a round cross section, a gauge diameter of $5 \mathrm{~mm}$ and gauge length of $19 \mathrm{~mm}$ were prepared using EDM. In order to help isolate the effects various deformation related phenomena the specimens were subjected to sub-yield cyclic loading in both the superelastic regime $15-750 \mathrm{MPa}$, as well as below the superelastic transition stress between 15-300 MPa. Furthermore, different numbers of load-unload cycles were investigated: 1, 2, 20 and 200 cycles at a loading rate of $4 \mathrm{MPas}^{-1}$. A $100 \mathrm{kN}$ Instron servo-hydraulic thermo-mechanical fatigue (TMF) frame was used to carry out the mechanical tests with a contact extensometer to record the macroscopic strain.

Subsequent to cyclic testing, the specimens were sectioned perpendicular to the loading axis for electron microscopy. Thicker sections were used to prepare specimens for electron backscatter diffraction (EBSD) mapping in the Zeiss Auriga field emission gun scanning electron microscope (FEG SEM) with an Oxford Instruments HKL Nordlys EBSD detector. Specimen surface preparation involved mechanical grinding using silicon 
carbide paper followed by polishing using colloidal silica suspension neutralised to $\approx \mathrm{pH} 7$ using hydrogen peroxide.

Thin slices were used to prepare specimens for transmission electron microscopy (TEM). Spark erosion was used to prepare $3 \mathrm{~mm}$ discs, which were then subjected to twin-jet electropolishing using an $8 \%$ solution of sulphuric acid in methanol at $18 \mathrm{~V}$ and $-40{ }^{\circ} \mathrm{C}$. Imaging of the resulting foils was performed using the JEOL JEM 2000FX $200 \mathrm{keV}$ thermionic emission TEM and the FEI Titan 80/300 FEG $C_{s}$ aberration corrected TEM/STEM at $300 \mathrm{keV}$.

\section{Results}

\subsection{Macroscopic stress-strain behaviour}

Figure 2 shows the stress vs. strain curves recorded during 200 load-unload cycles carried out on the TMF load frame. The first observation is the apparent accumulation of permanent plastic strain after every cycle, visible as gradual drift of the stress strain hysteresis greater residual strains. After 200 cycles, a total of approximately $0.2 \%$ strain had been accumulated by the specimen.

It can also be seen from the graph that the shape of the macroscopic behaviour changes progressively with every load cycle. For this, it is sufficient to compare the first and last load cycles. These have been highlighted by black dashed lines and are on the left and right respectively. It is clearly evident that the shapes of the two loops are quite different. There are three key observations that can be made:

The first loop confines a much larger area than the last. This implies that the material dissipates significantly more energy per unit volume during the first cycle. It is also worth noting that the overall shape of the first loop is different to those of all subsequent loops. It has a much broader shape when under high stresses. All subsequent loops are approximately three times narrower at their widest point and become progressively closer in shape to a classical hysteresis loop. This suggests that significant changes occur in the material during the first load cycle that alter its ability to accommodate strain during subsequent cycling.

The threshold stress for the superelastic transition is substantially reduced after 200 cycles. In the first loading curve the superelastic transition occurs at approximately $500 \mathrm{MPa}$. For comparison, this transition occurs at $\approx 200 \mathrm{MPa}$ in the final loading curve. This is a dramatic reduction which is evidence of permanent changes in the alloy that make the onset of superelastic behaviour more favourable when the load is reapplied.

Lastly, there is an apparent reduction in the Young's modulus of the specimen. The gradient of the elastic section of the loading curve drops from $\approx 65 \mathrm{GPa}$ in the first loading curve to $\approx 55 \mathrm{GPa}$ in the $200^{\text {th }}$ one.

All of these observations raise important questions about the extent of reversibility of the $\beta$ to $\alpha^{\prime \prime}$ martensitic transformation in Gum Metal. The alloy appears to accumulate permanent mechanical damage during load cycling within the limits of the superelastic regime. To better explain this behaviour and identify its mechanisms one must examine what happens in the material at the microscopic scale, which is described in the following two sections. 


\subsection{Synchrotron X-ray scattering}

Figure 3 shows the evolution of the (Intensity vs. $2 \theta$ ) diffraction spectrum during tensile loading of Gum Metal in the first cycle. As the applied tensile stress is increased past the superelastic transition from 15 to $700 \mathrm{MPa}$, Figure 3(a), we can observe appearance and gradual rise in prominence of two distinct diffraction peaks. These peaks correspond to the $\{110\}$ and $\{132\} \alpha^{\prime \prime}$ reflections respectively. Thus, our experiments show the same $b c c$ to orthorhombic diffusionless phase transformation that has been described by Talling et al. $[3,13]$.

Upon removal of the applied stress, Figure 3(b), the diffraction peaks attributed to the $\alpha^{\prime \prime}$ martensite subsided considerably. This was always found to be the case, irrespective of the number of load cycles that the specimen was subjected to. Therefore, any $\alpha^{\prime \prime}$ phase that may be retained after removal of the load must occupy an insignificant volume fraction and therefore is unlikely to be the source of the observed damage accumulation in the material. Figure 3 (c), shows the evolution of the fitted area of the $\{132\} \alpha^{\prime \prime}$ peak during loading and unloading in the first cycle. We observe that the evolution of the peak area also exhibits hysteresis and that the final peak area after unloading is smaller (i.e. near zero) than that prior to loading. This indicates the $\alpha^{\prime \prime}$ that existed within the diffracting before the application of stress has reverted back to $\beta$ on completion of the first cycle. Nevertheless, the superelastic transformation itself must play a significant role. In order to better understand the observed phenomena, we now turn to examine the changes that take place in the matrix phase, $\beta$.

Taking into account the texture resulting from extrusion, the $\{110\},\{211\}$ and $\{220\}$ reflections were analysed since they all have a component along the loading axis. They are persistent, considerably more intense than the $\alpha^{\prime \prime}$ peaks and allow for comparatively straightforward and reliable fitting. Figure 4 shows the evolution of the peak position for these reflections for selected load-unload cycles. The peak positions also exhibit hysteretic behaviour and show similar changes to those observed in the macroscopic stress vs. strain curve in Figure 2. Here one can see the same drastic change in the shape of the curve from cycle 1 to cycle 2 . In particular, there the same change in the width of the hysteresis. Figure 5(c) shows how the hysteresis width of the $\{110\}$ peak is reduced during superelastic load cycling. The rate of reduction is comparatively great at the beginning and becomes more gradual after approximately 5 cycles. The reduction in the threshold stress for the superelastic transformation can also be observed.

In addition, the $\{211\}$ peak shows peculiar behaviour. In the case of the first cycle, an apparently compressive strain is permanently imparted to the system after loading and unloading. The overall shape of the stress vs. strain loop is similar to the first cycles for $\{110\}$ and $\{220\}$, but it is reflected in the vertical axis and rotated $90^{\circ}$ about the origin. While we observe that none of the load-unload cycles for the three peaks formed closed loops, the first cycle of the the $\{211\}$ peak shows very clearly the most noticeable difference between the initial and final strain values. This is yet more evidence of permanent changes in the material. Closure of this substantial gap would require a compressive stress. Interestingly, during subsequent load-unload cycles the behaviour is similar to that exhibited by the $\{110\}$ and $\{220\}$ peaks.

Lastly, hysteresis drift can be observed in the positions of the $\{211\}$ and $\{220\}$ peaks, which appear to accumulate compressive and tensile strain respectively. The $\{110\}$ peak 
position also exhibits accumulation of tensile strain after twenty load-unload cycles, though this change is less noticeable than that those of the other two peaks.

Other properties of the three diffraction peaks, $(\{110\},\{211\}$ and $\{220\})$, also change during load cycling and provide additional evidence of mechanical damage accumulation. Figure 5(a) shows that the fitted width of the three peaks increases with each subsequent load-unload cycle. This increase is gradual for the $\{110\}$ and $\{220\}$ widths. In comparison, the behaviour of the $\{211\}$ is very peculiar, showing quite a rapid increase in width during the first 5-6 load-unload cycles. After that, the width continues to increase gradually at a similar rate to the other two.

The diffraction elastic constants (DECs) measured using lattice strains also show a decreasing trend, Figure 5(b). (A DEC is defined by the gradient of the initial microscopic stress with respect to the measured elastic lattice strain for a given lattice plane or family of lattice planes. In this study DECs were evaluated between 20 and $200 \mathrm{MPa}$.) The $\{110\}$ and $\{220\}$ DECs show a large drop after the second cycle. After that, they decrease linearly at a slower rate. In contrast, the $\{211\}$ stands out again. The initial dramatic drop in the DEC is spread over the first 5-6 cycles. This is then followed by a slight increase until cycle 10, after which the DEC decreases at approximately the same rate as the $\{110\}$ and $\{220\}$.

The results of the X-ray scattering experiment strongly suggest that significant structural changes (i.e. so called mechanical damage) take place in the Gum Metal specimen during superelastic load cycling which lead to the observed behaviour. The results suggest that the $\alpha^{\prime \prime}$ martensite transforms back to $\beta$ when the sample is unloaded. Thus, the mechanisms of the damage accumulation must take place at very fine length scales. To shed some light on this, we performed the following examination of the microstructure in the extruded material and load-cycled specimens.

\subsection{Electron microscopy}

The extruded bar was sectioned, polished using colloidal silica suspension, etched using $8 \mathrm{vol} . \% \mathrm{HF}$ and $15 \mathrm{vol} . \% \mathrm{HNO}_{3}$ in water and examined in a visible light microscope. Sections taken perpendicularly to the extrusion direction showed extensive 'marbling' that is characteristic of cold-deformed $b c c$ material. This indicates that the microstructure is not fully recrystallised after extrusion. It also suggests that not all of the plastic deformation sustained during the extrusion process has been recovered.

Figure 6 shows the results of the EBSD analysis performed on the un-cycled Gum Metal bar normal to the extrusion direction. The analysis shows that the material exhibits a strong texture, with the $\langle 110\rangle$ crystallographic axes of the grains aligned to the extrusion direction, Figure 6(b) and (c). More interestingly however, the composite band contrast and Euler angle map in Figure 6(a) shows the nature of the 'marbling' effect. The highly textured grains appear to form clusters in which their crystallographic misorientation relative to one another is small. Indeed, TEM shows that many grains are often separated by low-angle twist or tilt boundaries. A characteristic example boundary can be seen in the top right hand corner of Figure 7(a). Thus, crystallographic defects may propagate more easily between the grains within these domains.

Bright field TEM overviews of the dislocation content in the extruded and 200 cycle specimens are presented in Figure 7. From Figure 7(a) one can see that the extruded ma- 
terial already contains a population of dislocations. These are observed either as isolated segments or as networks. No significant changes to the arrangement and distribution of dislocations were observed in the specimen that was subjected to only one load-unload cycle.

However, after 200 cycles, we observe not only an apparent increase in the number density of dislocations, but also the formation of distinct slip bands, Figures 7(b) and (c). These bands are not observed in the un-cycled material, neither are they present after the first load cycle. This indicates that the formation and growth of the slip bands is a a gradual process that leads to the accumulation of plastic strain during load cycling. Thus, it is the most likely explanation for the hysteresis drift observed in Figure 2.

The gradual broadening of the diffraction peaks in Figure 5(a) is also consistent with this observation. The crystal lattice is distorted by a dislocation core, whereby it is either under compression or tension. Thus, accumulation of plastic strain can be observed as the broadening of the diffraction peaks in X-ray and neutron scattering experiments [14]. However, while all three of the diffraction peaks Figure 5(a) exhibit broadening, the most dramatic change takes place in the width of of the $\{211\}$ peak. Furthermore, this change takes place over the first few cycles. This implies that either there is another deformation related phenomenon contributing to the broadening, or that the number density of dislocations causing the broadening increases tremendously during the first few load-unload cycles.

Figure 8 illustrates the effect of superelastic load-cycling on the distribution of the $\omega$ phase precipitates. (Both dark field images taken using the $\omega$ satellite spots near the $\{110\}$ zone axis and the corresponding diffraction patterns are shown.) In Figure 8(a), we see that the material in the as extruded condition already has a population of fine $\omega$. However, it should be noted that this distribution is quite uneven and grains still exist with hardly any precipitates at all. In other instances, the $\omega$ phase can be found concentrated in isolated pockets near grain boundaries. This may be a consequence of the hot extrusion process, which results in the highly deformed and textured microstructure, as is evident in Figure 6.

Subjecting the Gum Metal to a single load-unload cycle appears to increase the fraction of the $\omega$ phase precipitates, as well as their apparent size, Figure 8(b). This is evident in both the dark field image and the diffraction pattern, where the $\omega$ satellite spots are considerably brighter. This observation is consistent with the finding of Jones et al. [8], who also observed this trend. In contrast with the un-cycled material, the $\omega$ phase was also more evenly distributed throughout the TEM specimen. After 200 cycles, we observe that the amount of the $\omega$ phase has increased further, Figure 8(c). The precipitates also appear to have either increased in their average size, or to have formed distinct clusters.

The observed formation of $\omega$ phase during the first load-unload cycle coincides with a change in the shape of the hysteresis loop that is evident between the first and second cycles. Thus, it is likely that the superelastic behaviour is affected by the precipitation of $\omega$. Our observations suggest an increase in the $\omega$ fraction correlates with the gradual decrease in the threshold stress for the superelastic transition. It is possible that the presence of $\omega$ somehow facilitates the martensitic phase transformation responsible for superelastic behaviour. It should also be noted that the formation of slip bands may also be contributing to this phenomenon. We therefore propose that a detailed study is carried out using small angle neutron/X-ray scattering (SANS/SAXS) to asses the dependence of the $\omega$ volume fraction and size distribution on superelastic load cycling. 
Twinning of the $b c c \beta$ phase is also observed in specimens that have been subjected to 200 load-unload cycles. The twinned structures are of two types: solitary laths and colonies. Examples of the solitary twins imaged close to the $\{110\}$ zone axis are shown in Figure $9(\mathrm{a}-\mathrm{d})$. They have thicknesses ranging from $\approx 5$ to $50 \mathrm{~nm}$ and their ends are tapered to a point, Figure 9(d). Such morphology is characteristic of deformation twins [15]. Figure 9(e) shows a pseudo diffraction pattern (frequency domain image) generated using a fast Fourier transform (FFT) of the high-resolution lattice image in Figure 9(c). (N.B. Figure 9(b) shows the same twin at lower magnification.) The spot pattern confirms that the structure is indeed twinned $\beta$ phase. Figure $9(\mathrm{f})$ is a schematic of the pattern in (e), where the red and pink spots correspond to the material within the lath and black and grey spots to the material that surrounds it. From the lattice images and diffraction patterns in Figure 9 it is possible to deduce that the interface plane of the twin and the surrounding material is close to the $\{112\}$. It is peculiar that these deformation twins are only observed in grains that feature slip bands, and are typically found within the slip bands themselves running parallel to them, as can be seen in Figure 9(a). This suggests that one type of defect structure may act as the source of the other.

Figure 10 shows an example of a twin colony found in a specimen that was subjected to 200 load-unload cycles. Due to the bending of the TEM specimen, the diffraction contrast bright field image in 10(a) reveals very well the structure of the colony. One can see smaller twins within larger twined regions. Figure 10(b) shows, at greater magnification, the structure of the finer twins in the region marked by the square in Figure 10(a). The six twins visible in the image have been numbered from left to right. The region imaged in Figure 10(b) is aligned to a zone axis (visible from the bend contours in Figure 10(a)) phase contrast imaging of the lattice is possible. Figure 10(c) shows the lattice structure of twin 5 and the interface with the surrounding twins. The banding of the interface results from the interface planes not being normal to the image plane, causing slight overlap of the twinned crystal in the image.

Figure 10(d) shows the FFT frequency domain images of twinned regions 1-4. The spot patterns indicate that the viewing direction in Figure 10(c) is parallel to the $\{012\}$ zone axis of the $b c c \beta$ phase and that the observed structures are indeed twins. The measured average values of $d_{\{002\}}=1.650 \AA$ and $d_{\{112\}}=1.345 \AA$ (to 4 significant figures) give the corresponding values for the lattice constant of $3.300 \AA$ and $3.295 \AA$. These fit well with the lattice parameter measured using synchrotron X-ray diffraction, 3.2907 $\AA$. The values measured from the electron image may be larger due to heating imparted on the sample by the electron beam. Diffuse streaking and interference spots can be distinguished in the frequency domain images, particularly well for twin 3 . These are likely to correspond to the abundant $\omega$ phase. The particular morphology of the twins suggests that they may have originated via the reverse transformation of the twinned $\alpha^{\prime \prime}$ phase back to the $b c c$ $\beta$ phase upon unloading.

\section{Discussion}

The results of our study show that load cycling of Gum Metal in the superelastic regime induces permanent microstructural changes. Furthermore, the extent and nature of these changes depends on the number of load cycles sustained by the material. In order to better understand the causes of the observed phenomena, we now consider the nature of the 
superelastic transformation in Gum Metal. For this, we can employ the phenomenological theory of martensitic transformation (PTMT) as outlined by Lieberman, Wechsler and Read [16,17] and Mackenzie and Bowles [18-21] to model the cubic to orthorhombic transformation of $\beta$ to $\alpha^{\prime \prime}$.

The theory allows the prediction of the interface plane, orientation relationships and macroscopic distortions for the transformation using only the lattice parameters of the austenite and martensite phases as an input. It shows that an interface plane with zero distortion (i.e. an invariant plane) minimises the strain energy associated with the transformation. This is achieved through the twinning of the martensite phase in such a way where the relative amounts of the two twin variants, $x_{1}$ and $x_{2}$ where $x_{1}=1-x_{2}$, satisfy this condition.

In our calculation we used the following lattice parameters which were measured experimentally during the in-situ synchrotron experiment: $a_{\beta}=3.347 \AA, a_{\alpha^{\prime \prime}}=3.225 \AA$, $b_{\alpha^{\prime \prime}}=4.763 \AA, c_{\alpha^{\prime \prime}}=4.636 \AA$. For comparison, the values reported by Talling et al. [3] were also subjected to the analysis $\left(a_{\beta}=3.347 \AA, a_{\alpha^{\prime \prime}}=3.250 \AA, b_{\alpha^{\prime \prime}}=4.853 \AA, c_{\alpha^{\prime \prime}}=4.740 \AA\right)$. All results are reported relative to the cubic crystal coordinate system of the parent $\beta$ phase. It should also be noted that left-handed coordinate axes were used, following the methodology of the original manuscript by Lieberman et al. [16].

According to the their approach the total distortion $\mathbf{E}$ caused by the transformation is given by the following expression:

$$
\mathbf{E}=\left(1-x_{2}\right) \boldsymbol{\Phi}_{\mathbf{1}} \mathbf{T}_{\mathbf{1}}+x_{2} \mathbf{\Phi}_{\mathbf{2}} \mathbf{T}_{\mathbf{2}}
$$

The matrices $\mathbf{T}_{\mathbf{1}}$ and $\mathbf{T}_{\mathbf{1}}$ describe the Bain $[22,17]$ distortion for each martensite variant.

In the cubic (austenite) reference frame these these were defined as

$$
\mathbf{T}_{\mathbf{1}}=\left(\begin{array}{ccc}
\eta_{1} & \eta_{2} & 0 \\
\eta_{2} & \eta_{1} & 0 \\
0 & 0 & \eta_{3}
\end{array}\right) \text { and } \mathbf{T}_{\mathbf{2}}=\left(\begin{array}{ccc}
\eta_{1} & 0 & \eta_{2} \\
0 & \eta_{3} & 0 \\
\eta_{2} & 0 & \eta_{1}
\end{array}\right)
$$

where $\eta_{1}=\sqrt{2}\left(b_{\alpha^{\prime \prime}}+c_{\alpha^{\prime \prime}}\right) / 4 a_{\beta}, \eta_{2}=\sqrt{2}\left(-b_{\alpha^{\prime \prime}}+c_{\alpha^{\prime \prime}}\right) / 4 a_{\beta}$ and $\eta_{3}=a_{\alpha^{\prime \prime}} / a_{\beta}$. The rotations necessary to attain a zero distortion plane are given by the matrices $\boldsymbol{\Phi}_{1}$ and $\boldsymbol{\Phi}_{2}$. The PTMT first calculates the relative rotation between $\boldsymbol{\Phi}_{1}$ and $\boldsymbol{\Phi}_{2}$. This allows the relative amounts of the twin variants, $x_{1}$ and $x_{2}$, that are necessary to attain an invariant plane to be determined along with the principal distortion matrix, $\mathbf{F}_{\mathbf{d}}$, where:

$$
\mathbf{F}_{\mathbf{d}}=\left(\begin{array}{ccc}
\lambda_{1} & 0 & 0 \\
0 & \lambda_{2} & 0 \\
0 & 0 & \lambda_{3}
\end{array}\right)
$$

To satisfy the invariant plane condition, one of the principal distortions, $\lambda_{i}$, must be unity

when the correct proportions of the twin variants, $x_{1}$ and $x_{2}$, are formed. This condition allows one to calculate the solution using linear algebra. This in turn allows the total distortion, E, to be determined and with it the critical parameters for the transformation 
including orientation relationship between the austenite and martensite phases are also found.

Table 1 lists these key properties of the transformation where $\mathbf{n}$ is the normal to the invariant interface plane; $\mathbf{s}, m$ and $\theta$ are the direction, magnitude and angle of shear respectively; while $\mathbf{t}$ is the twin plane between the two martensite variants. The rotation matrices $\Theta$ and $\boldsymbol{\Omega}$ transform the cubic planes and directions to the orthorhombic axis systems of the martensite variants 1 and 2 respectively. Their components evaluated for the lattice parameters measured in this study are shown below and the corresponding orientation relationships are given in Table 2.

$$
\begin{aligned}
& \boldsymbol{\Theta}=\left(\begin{array}{rrr}
0.7190 & -0.7039 & 0.0108 \\
0.6851 & 0.6994 & -0.0255 \\
0.0102 & 0.0252 & 0.9632
\end{array}\right)_{\beta \rightarrow \alpha_{1}^{\prime \prime}} \\
& \boldsymbol{\Omega}=\left(\begin{array}{rrr}
0.7136 & -0.0414 & -0.7083 \\
0.0025 & 0.9620 & -0.0538 \\
0.6906 & 0.0370 & 0.6936
\end{array}\right)_{\beta \rightarrow \alpha_{2}^{\prime \prime}}
\end{aligned}
$$

Below are the matrices evaluated using lattice parameters measured by Talling et al. and the orientation relationships are shown presented in Table 3.

$$
\begin{aligned}
& \boldsymbol{\Theta}=\left(\begin{array}{rrr}
0.7452 & -0.7028 & -0.0438 \\
0.6877 & 0.7273 & 0.0309 \\
0.0095 & -0.0503 & 0.9697
\end{array}\right)_{\beta \rightarrow \alpha_{1}^{\prime \prime}} \\
& \boldsymbol{\Omega}=\left(\begin{array}{rrr}
0.7268 & 0.0192 & -0.7229 \\
-0.0165 & 0.9708 & 0.0092 \\
0.7061 & 0.0052 & 0.7101
\end{array}\right)_{\beta \rightarrow \alpha_{2}^{\prime \prime}}
\end{aligned}
$$

One can see from these data that the interface and twin planes, $\mathbf{n}$ and $\mathbf{t}$ respectively, are irrational for both sets of lattice parameters. The implication of this is that these interfaces are likely to be semi coherent and feature a stepped geometry that is stabilised by the presence of dislocations. This hypothesis is consistent with the experimental evidence presented in our study since the superelastic transformation results in the creation of new interfaces feature lattice defects that lead to an irreversible accumulation of strain. That said, the planarity and coherence of these interfaces is difficult to assess: HR TEM is sensitive to specimen bending (which is prevalent in foils prepared from highly deformed Gum Metal) and the averaging effect associated with imaging columns of atoms also plays a part. Furthermore, no $\alpha^{\prime \prime}$ martensite phase is retained in our specimens that would permit a direct evaluation.

The differences between the the results for the two sets of lattice parameters are also of interest. The lattice parameters measured here require two principal distortions that 
are both smaller than unity, which implies that both are contractions. When using the parameters of Talling et al., one of the distortions is greater that unity and one is smaller, with the implication that one is an expansion and the other is a contraction. The overall magnitude of shear is also greater for this study. Lastly there is difference in the relative amounts of the two twin variants. These discrepancies may be a result of different techniques used to measure the lattice parameters (Synchrotron XRD vs. electron diffraction). However, the parameters reported by by Talling et al. are actually average values for alloys with different compositions: Ti-36.9Nb-2.0Ta-3.0Zr-0.30O and Ti$35.0 \mathrm{Nb}-2.1 \mathrm{Ta}-3.1 \mathrm{Zr}-0.30 \mathrm{O}$ wt.\%. The Gum Metal used in this study has a lower oxygen content $(0.26$ wt.\%). This may contribute to the difference in observed lattice parameters and the PTMT calculation results, since oxygen concentration is known to have a strong effect on the martensitic transformation and $\omega$ phase formation in $\beta$ titanium alloys $[13,10]$.

The mathematical transformation theory seems to provide a possible explanation for the formation for the increased dislocation density observed as slip bands in Figure 7 . The glide planes of the dislocation segments making up the slip bands are close to the $\{112\}$ habit planes of the deformation twins. The interface plane normals, $\mathbf{n}$, calculated using PTMT are irrational. Their normals have a misorientation from $(1 \overline{2} \overline{1})_{\beta}$ of $\approx 10.0^{\circ}$ and $8.0^{\circ}$ respectively for the two sets of lattice parameters. It is possible that the necessary stepped geometry which is required for the high index irrational interface planes is accommodated by $\frac{a}{2}\langle 111\rangle\{112\}$ dislocations. Thus the observed slip bands may be formed at or within the vicinity of the $\beta / \alpha^{\prime \prime}$ interfaces as the material is subjected to superelastic load cycling. When the load is reapplied during each cycle, the remnant defects may favour the subsequent reformation of the interfaces close to their locations in the previous cycle. The application of the load is also likely to propagate the generated dislocations, further contributing to the increase in their number density over time.

This in turn sheds light on the origin of the deformation twins in close proximity or within the slip bands, as shown in Figure 9. The current understanding is that deformation twins are likely to nucleate and grow via defect assisted mechanisms $[15,23]$. We consider that the homogeneous nucleation of twinning dislocations is unlikely. The glide planes of the dislocation segments making up the slip bands are close to the $\{112\}$ habit planes of the deformation twins. This observation is in line with "slip band conversion" model put forward for b.c.c. metals by Mahajan [24], based on the theories of coupling between $\frac{a}{2}\langle 111\rangle\{112\}$ screw dislocation slip and twin nucleation [25-27].

The observations discussed thus far suggest that upon removal of the tensile load the $\alpha^{\prime \prime}$ phase generally de-twins and reverts back to the $\beta$ crystal structure. In contrast, Figure 10 shows twinning of a different nature in the $\beta$ phase. The twin plane is also $\{112\}$, so the observed microstructure is unlikely to have formed via the reverse transformation of the $\alpha^{\prime \prime}$ twins which have a twin plane that is close to $\{110\}_{\beta}$. This leaves two possible routes for the formation of the twinned $\beta$ microstructure. In the first, the microstructure was formed directly via the twinning of the b.c.c. phase. Alternatively, if under load the preceding structure was comprised of alternating layers of $\beta$ and $\alpha^{\prime \prime}$, it is possible that upon removal of the load the the martensite phase underwent a reverse transformation, but to a twin variant of the original $\beta$ phase. Thus, the interface $\beta / \alpha^{\prime \prime}$ interface planes would have become twin planes separating two variants of $\beta$. This may be possible because of the relative proximity of the interface plane to $\{112\}_{\beta}$.

It is also worth noting that the calculated $\beta / \alpha^{\prime \prime}$ interface plane normals have a mis- 
orientation of $\approx 9.4^{\circ}$ and $12.8^{\circ}$ (respectively for the two sets of lattice parameters) from $(1 \overline{1} \overline{1})_{\beta}$, which is the habit plane of the $\omega$ phase. Thus, its possible that the precipitates of this phase also help accommodate the irrational interface between the austenite and martensite phases. Our observations show that load cycling produces more $\omega$ phase.

Thus, both our observations and the classical transformation theory show that defects are likely to form during the martensitic that gives rise to superelasticty. The generated defects appear to better accommodate the $\beta / \alpha^{\prime \prime}$ interface and thus lower the activation strain energy required for the martensitic transformation. This is the likely cause of the of the gradual reduction in the threshold stress for the transformation, as well as the overall energy dissipated in the hysteresis, observed in Figure 2.

\section{Conclusions}

In our study we have presented experimental evidence of defect accumulation that occurs during tensile superelastic load cycling of Gum Metal, which not only imparts a permanent plastic strain on the material, but also alters its superelastic hysteresis. The observed defects included dislocations forming distinct slip bands, deformation and transformation twins were also present and there was a transformation of the $\beta$ phase to fine athermal $\omega$ precipitates.

Synchrotron X-ray diffraction has identified peculiar behaviour of the $\{211\}$ diffraction peak when binned parallel to the tensile direction, that stood out from that of the $\{110\}$ and $\{220\}$ reflections. By carrying out a mathematical analysis of the $\beta$ to $\alpha^{\prime \prime}$ martensitic transformation using classical PTMT we have showed that the austenite/martensite interface plane is irrational and only $\approx 8$ to $10^{\circ}$ from the $\{211\}$. This suggests that the necessary steps in the interface are accommodated by the $\frac{a}{2}\langle 111\rangle\{112\}$ dislocations forming the slip bands observed in TEM. The slip bands conversion model then explains the formation of deformation twins on the $\{211\}$ planes. The $\beta / \alpha^{\prime \prime}$ interface plane normal is also $\approx 9$ to $13^{\circ}$ from the $\{111\}$, which suggests that the martensitic transformation and formation of athermal $\omega$ phase are also related.

The PTMT therefore provides a unified explanation for the origin of the observed defect structures and the resulting macroscopic behaviour. The gradual accumulation of defects has significant implications for exploiting the superelastic behaviour of Gum Metal, particularly in applications such as damping since the strain accumulation and changes in hysteresis (and thus energy dissipation characteristics) must be carefully taken into account. This may be overcome by fine tuning the alloy composition to produce an austenite/martensite interface that is closer to a rational low index plane, the formation of which would produce a minimal number of dislocations.

\section{Acknowledgements}

The authors would like to acknowledge financial support provided by the EPSRC under grant EP/H004882/1 - "Reducing Emissions by Exploiting Field-Induced Martensitic Transformations" (DD) and under the Doctoral Prize Fellowship Scheme (VAV). We recognise support from the DSTL MAST STC (KMR); the Diamond Light Source for funding experiment EE6701; Incotest, Hereford, UK for ICP-OES; Prof. Richard Dashwood, WMG, University of Warwick, UK for extrusion; Dr. Mahmoud Ardakani, Dept. 
of Materals, Imperial College London and Dr. Liam Spillane, Gatan UK, Abingdon, UK for assistance with electron microscopy; Dr. John Webster, Rolls-Royce plc, Derby, UK for valuable encouragement and discussion of the project.

\section{References}

[1] T. Saito, T. Furuta, J.-H. Hwang, S. Kuramoto, K. Nishino, N. Suzuki, R. Chen, A. Yamada, K. Ito, Y. Seno, T. Nonaka, H. Ikehata, N. Nagasako, C. Iwamoto, Y. Ikuhara, T. Sakuma, Science 300 (2003) 464-467.

[2] R. J. Talling, R. J. dashwood, M. Jackson, S. Kuramoto, D. Dye, Scripta Materialia 59 (2008) 669-672.

[3] R. J. Talling, R. J. Dashwood, M. Jackson, D. Dye, Acta Materialia 57 (2009) 1188-1198.

[4] J. W. Morris, Y. Hanlumyuang, M. Sherburne, E. Withey, D. C. Chrzan, S. Kuramoto, Y. Hayashi, M. Hara, Acta Materialia 58 (2010) 3271-3280.

[5] E. Plancher, C. C. Tasan, S. Sandloebes, D. Raabe, Scripta Materialia 68 (2013) 805-808.

[6] T. Yano, Y. Murakami, D. Shindo, S. Kuramoto, Acta Materialia 57 (2009) 628-633.

[7] T. Yano, Y. Murakami, D. Shindo, Y. Hayasaka, S. Kuramoto, Scripta Materialia 63 (2010) 536-539.

[8] N. J. Jones, R. J. Talling, T. C. Lindley, D. Dye, Failure behaviour and energy adsorption in Gum metal (Ti-36Nb-2Ta-3Zr-O), in: L. Zhou, H. Chang, Y. Lu, D. Xu (Eds.), Ti-2011, Science Press Beijing, 2012, p. 1169.

[9] P. Castany, M. Besse, T. Gloriant, Scripta Materialia 66 (2012) 371-373.

[10] M. Besse, P. Castany, T. Gloriant, Acta Materialia 59 (2011) 5982-5988

[11] E. Eisenbarth, D. Velten, M. Müller, R. Thull, J. Berne, Biomaterials 25 (2004) 5705-5713.

[12] A. P. Hammersley, S. O. Svensson, A. Thompson, Nuclear Instruments and Methods in Physics Research A 346 (1-2) (1994) 312-321.

[13] E. G. Obbard, Y. L. Hao, R. J. Talling, S. J. Li, Y. W. Zhang, D.Dye, R. Yang, Acta Materialia 59 (2011) $112-125$.

[14] N. G. Jones, D. Dye, Intermetallics 19 (2011) 1348-1358.

[15] J. W. Christian, S. Mahajan, Progress in Materials Science 39 (1995) 1-157.

[16] D. S. Lieberman, M. S. Wechsler, T. A. Read, Journal of Applied Physics 26 (1955) 473-484.

[17] M. S. Wechsler, D. S. Lieberman, T. A. Read, Transactions AIME 197 (1953) 1503-1515.

[18] J. S. Bowles, J. K. Mackenzie, Acta Metallurgica 2 (1954) 129-137.

[19] J. K. Mackenzie, J. S. Bowles, Acta Metallurgica 2 (1954) 138-147.

[20] J. S. Bowles, J. K. Mackenzie, Acta Materialia 2 (1954) 224-234.

[21] J. K. Mackenzie, J. S. Bowles, Acta Metallurgica 5 (1957) 137-149.

[22] E. C. Bain, Transactions AIME 70 (1924) 25-46.

[23] S. Mahajan, D. F. Williams, International Metallurgical Reviews 18 (1973) 43-58.

[24] S. Mahajan, Acta Metallurgica 23 (1975) 671-684.

[25] A. W. Sleeswyk, Philosophical Magazine 8 (1963) 1467-1486.

[26] K. Ogawa, Philosophical Magazine 11 (1965) 217-233.

[27] V. Vitek, R. C. Perrin, D. K. Bowen, Philosophical Magazine 21 (1970) 1049-1073.

[28] N. G. Jones, C. M. Ward-Close, P. M. Brown, D. Dye, Scripta Materialia 63 (2010) 85-88. 


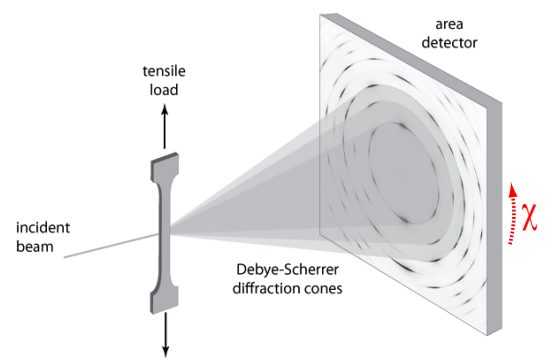

Fig. 1. Schematic representation of the experimental arrangement used to make the in-situ synchrotron measurements. The data are binned over an azimuthal angle, $\chi$, to produce intensity vs. $2 \theta$ spectra. Adapted from [28].

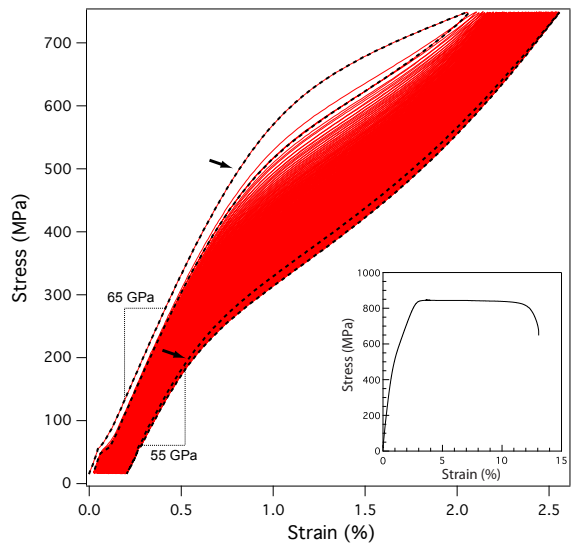

Fig. 2. Evolution of the stress vs. strain hysteresis over 200 load-unload cycles between 15-750 MPa. The loops corresponding to the first (left) and last (right) load cycles are highlighted by the black dashed lines. Arrows show approximate positions of superelastic transition stress upon loading. The full stress vs. strain curve for Gum Metal is shown in the bottom left corner. 

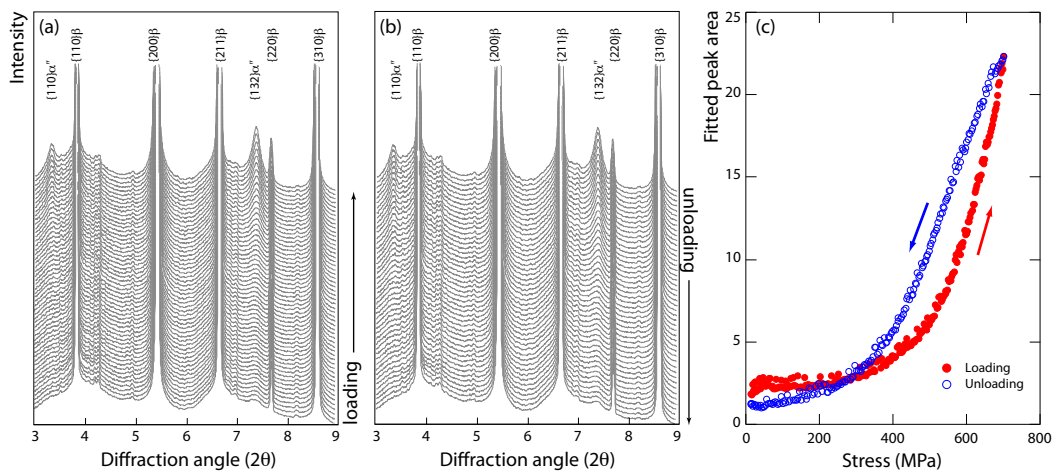

Fig. 3. X-ray scattering spectra for the first load-unload cycle showing the behaviour of $\alpha^{\prime \prime}$ scattering peaks amidst those produced by the $\beta$ phase with increasing (a) and decreasing (b) applied tensile stress (from 15 to $750 \mathrm{MPa}$ ). Subfigure (c) shows the evolution of the $\{132\}_{\alpha^{\prime \prime}}$ fitted peak area during the first cycle.

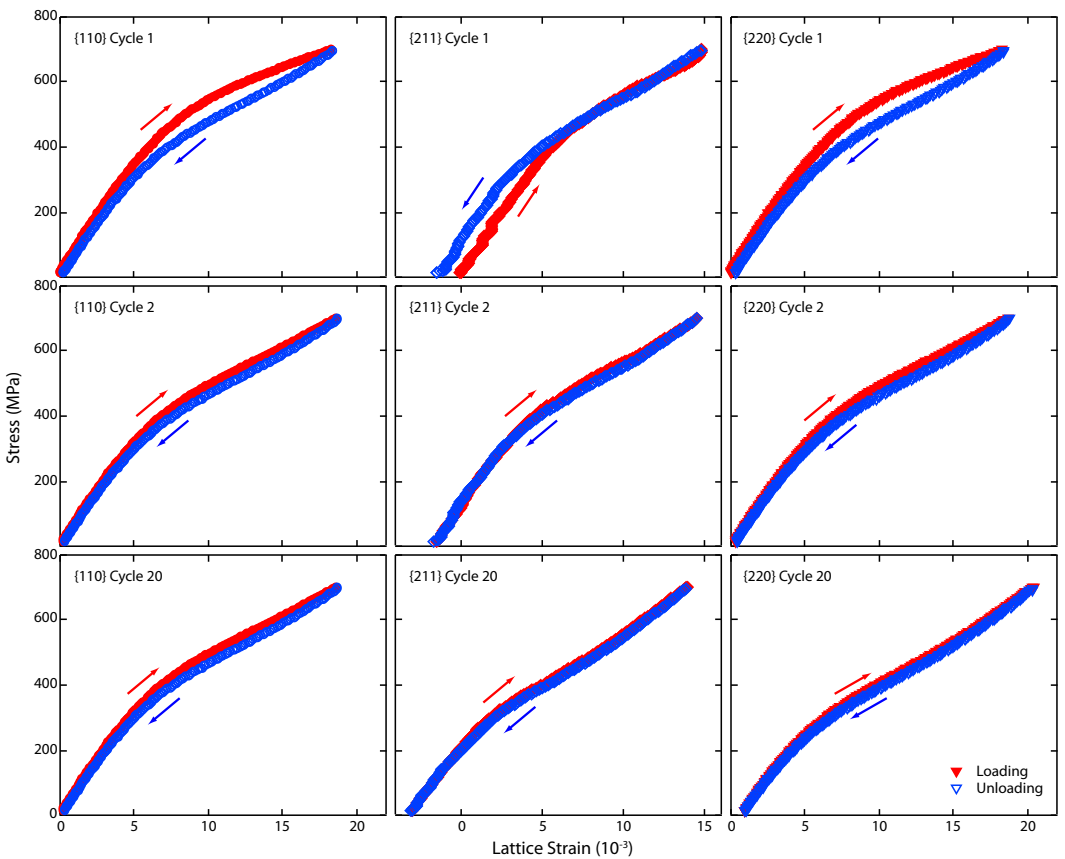

Fig. 4. Evolution of the positions of the $\beta$ phase $\{110\},\{211\}$ and $\{220\}$ X-ray diffraction peaks, all of which have a component along the tensile axis due to the strong texture of the test specimens. 

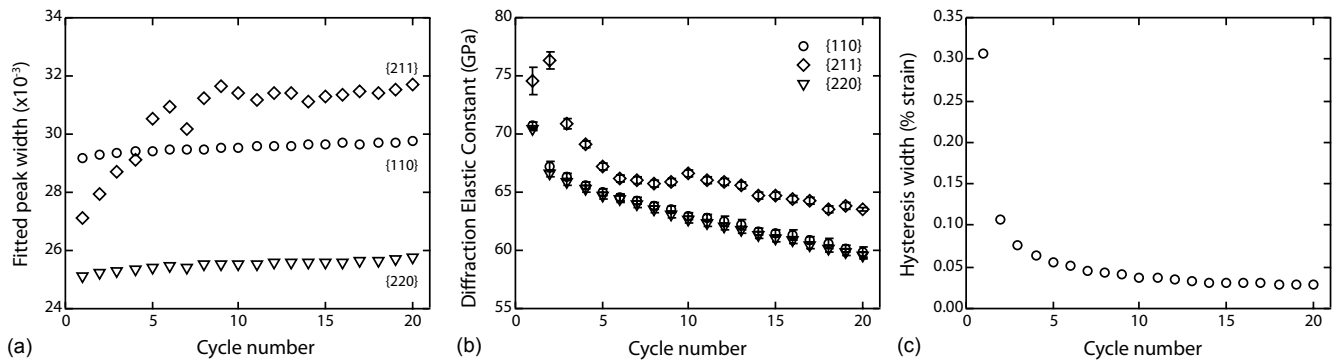

Fig. 5. Changes in the diffraction data of Gum Metal resulting from load cycling in the superelastic regime: (a) broadening of diffraction peaks with components binned parallel to the tensile axis, (b) gradual reduction in the diffraction elastic constants, (c) reduction in the width of the hysteresis loop of the $\{110\}$ peak taken at the end of each cycle when the sample had been unloaded to $15 \mathrm{MPa}$ and all of the $\alpha^{\prime \prime}$ had reverted.
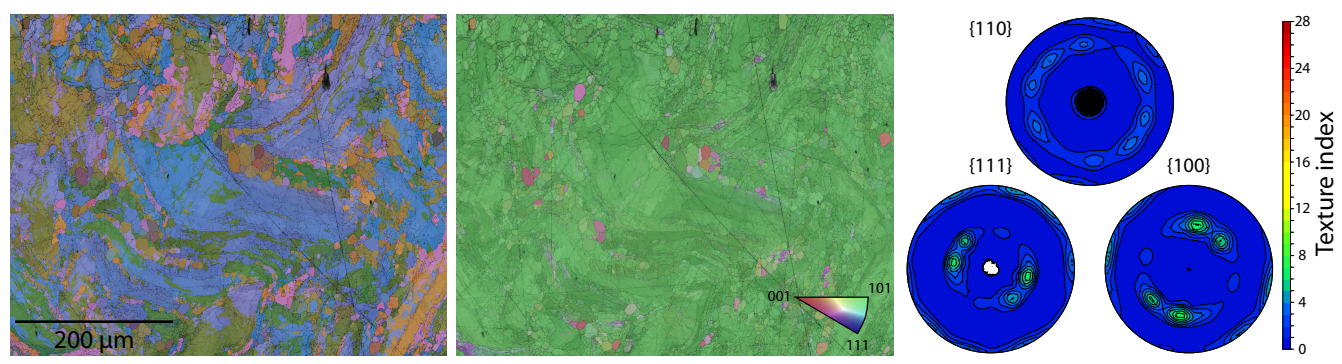

Fig. 6. EBSD analysis of microstructure and texture of the Gum Metal stock normal to the extrusion direction: (a) Euler angle map combined with a band contrast image,(b) inverse pole figure map combined with a band contrast image and (c) pole figures for the $\{100\},\{110\}$ and $\{111\}$ directions.
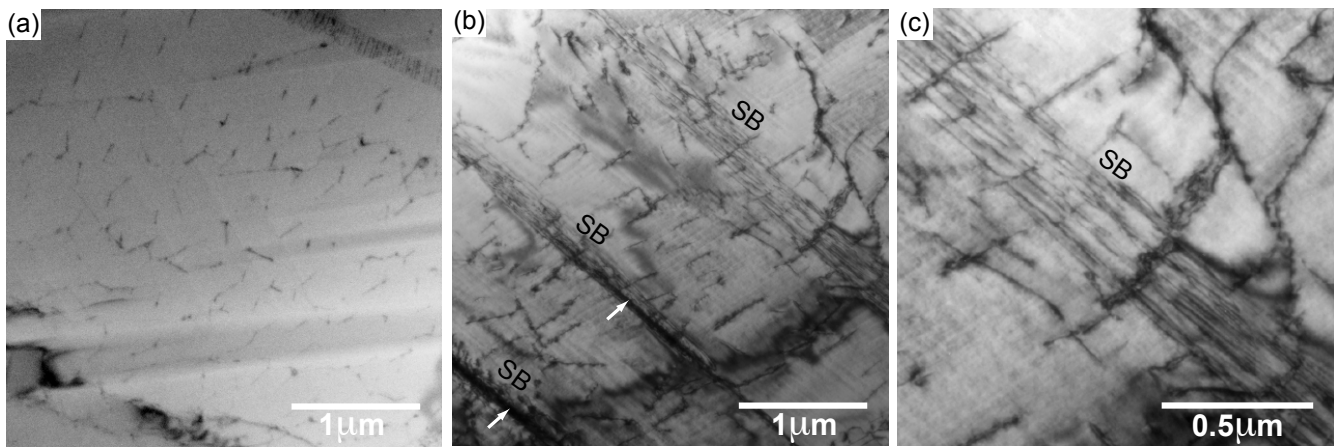

Fig. 7. Diffraction contrast bright-field TEM images showing a comparison of dislocation distributions in the extruded condition (a) and after 200 load-unload cycles (b) and (c). Slip bands have been labelled SB and white arrows point to the locations of deformation twins. 

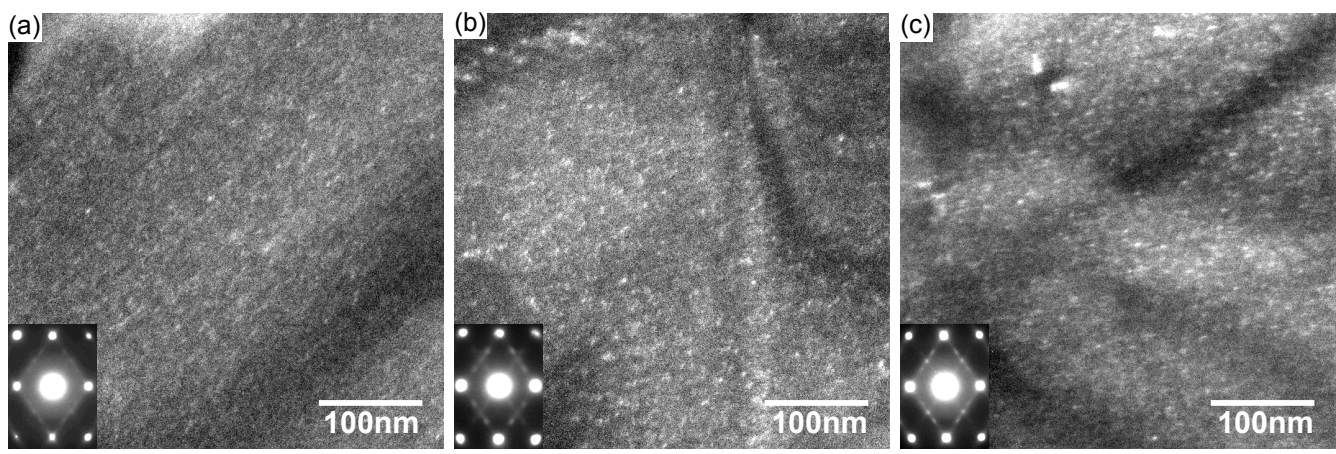

Fig. 8. Dark field TEM micrographs showing that superelastic load cycling increases the amount of the $\omega$ phase in Gum Metal: (a) as extruded condition, (b) 1 cycle and (c) 200 cycles.
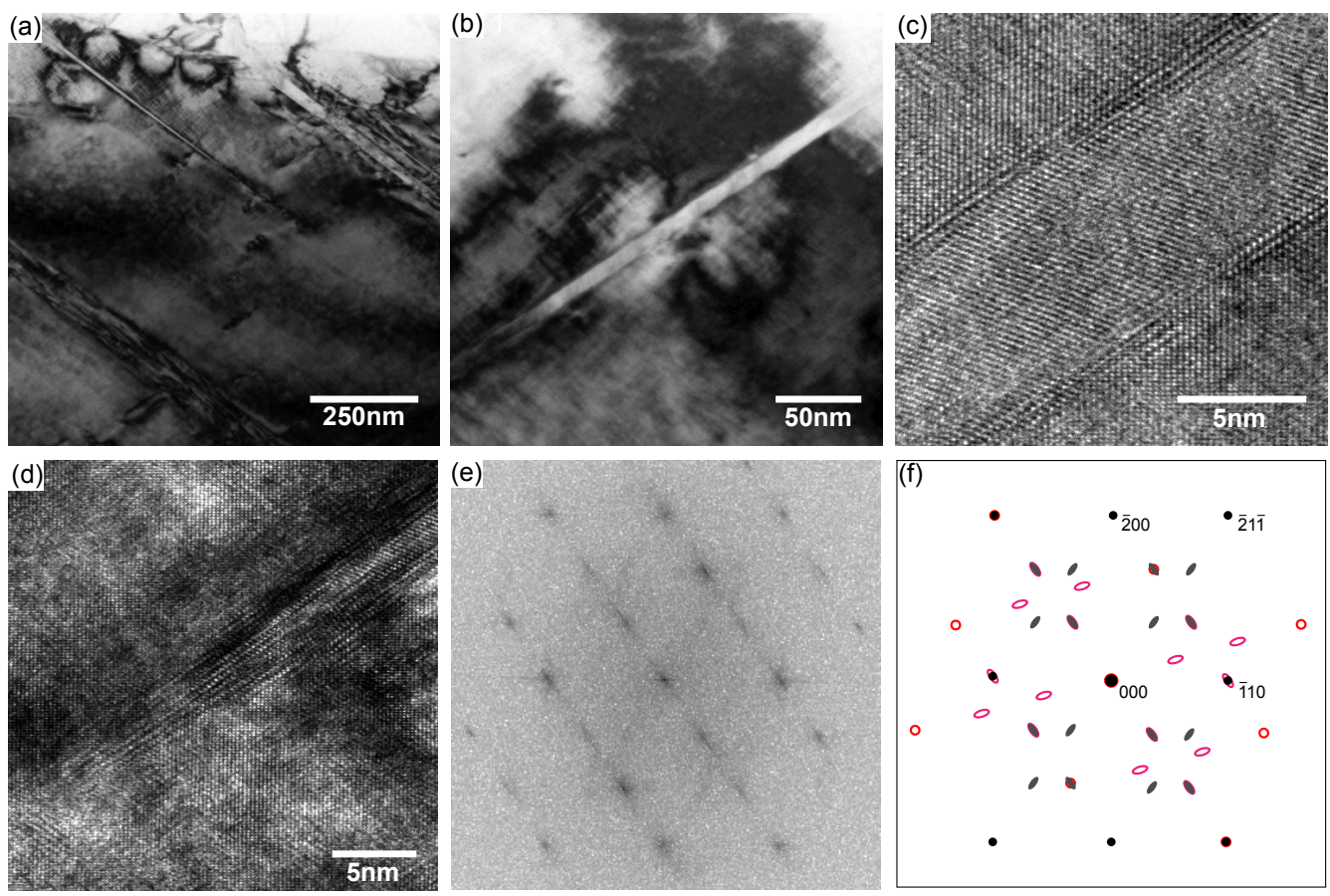

Fig. 9. Diffraction contrast bright field TEM images of fine deformation twins observed after 200 load-unload cycles, (a) and (b). High-resolution phase contrast lattice imaging showing the structure of the twin in the middle (c) and at the tip (d). Frequency domain image (e) obtained using FFT of lattice image (c) and the corresponding schematic denoting showing contributing spot patterns from the twin (red) and the surrounding matrix (black). 

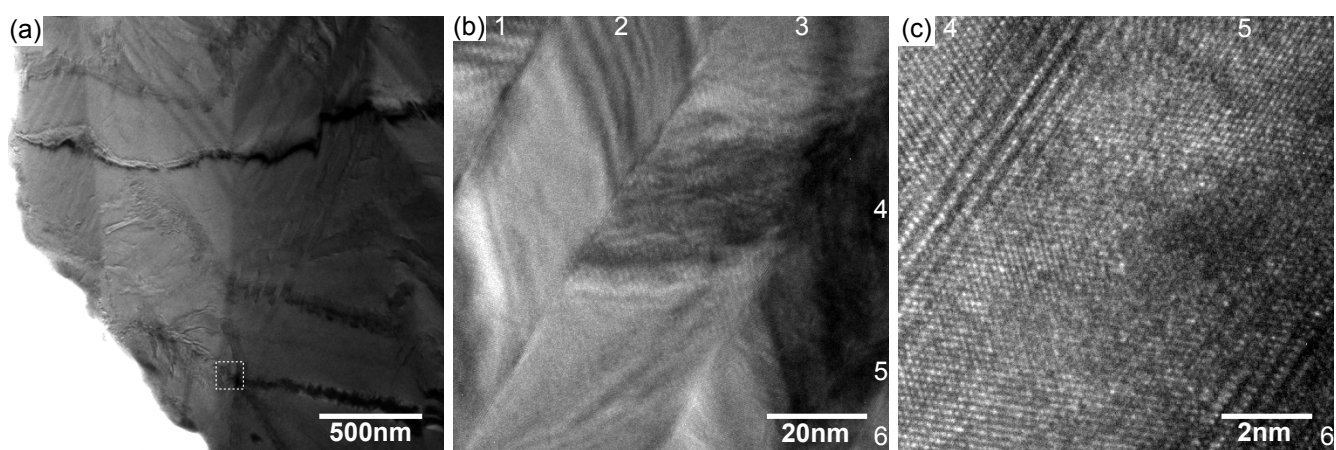

(d)
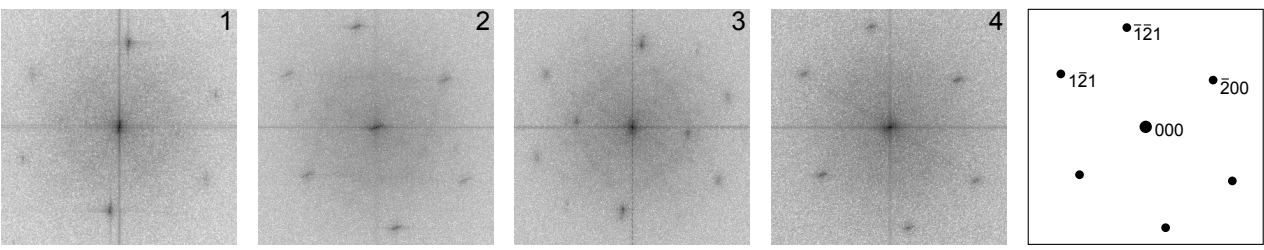

Fig. 10. Diffraction bright field TEM image of a twin colony observed after 200 load-unload cycles, (a) and high-resolution phase-contrast close-up (b) of region marked by the white square in (a). High-resolution phase contrast lattice image (c) of twin 5 in (b). Frequency domain images (d) of obtained using FFT of the lattice in twins 1-4 in (b) and corresponding schematic denoting the Miller indices of the spots in 4 .

Table 1

Key properties of the $\beta \rightarrow \alpha^{\prime \prime}$ martensitic transformation calculated using PTMT [16]. The top and bottom sets were computed using lattice parameters measured in this study (top) and by Talling et al. [3] (bottom). The parameters, from left to right respectively, are proportions of the $\alpha^{\prime \prime}$ twins, $x_{1}$ and $x_{2}$, distortion matrix, $\mathbf{F}_{\mathrm{d}}$, interface plane normal, $\mathbf{n}$, direction of shear, s, magnitude of shear, $m$, angle of shear, $\theta$ and $\alpha^{\prime}$ twin plane, t. All vectors are quoted with respect to the cubic coordinate axes of the $\beta$ phase.

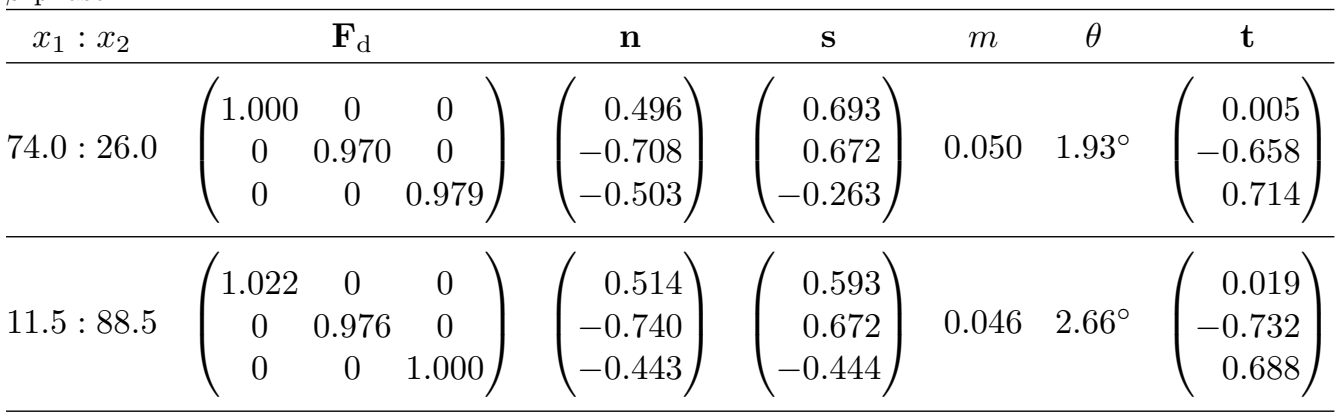


Table 2

Orientation relationships obtained using lattice parameters measured in this study.

\begin{tabular}{|c|c|}
\hline \multirow{2}{*}{\multicolumn{2}{|c|}{$\frac{\beta \text { and } \alpha^{\prime \prime} \text { twin } 1}{\beta \text { and } \alpha^{\prime \prime} \text { twin } 2}$}} \\
\hline & \\
\hline$(10 \overline{1})_{\beta} 1.61^{\circ}$ from $(010)_{\alpha_{1}^{\prime \prime}}$ & ${ }^{\prime \prime}(010)_{\beta} 3.20^{\circ}$ from $(010)_{\alpha_{2}^{\prime \prime}}$ \\
\hline$(001)_{\beta} 1.61^{\circ}$ fror & $1)_{\beta}$ \\
\hline$[111]_{\beta} 1.71^{\circ}$ from $[011]_{\alpha_{1}^{\prime \prime}}$ & {$[111]_{\beta} 2.48^{\circ}$ from $[011]_{\alpha}$} \\
\hline$[11 \overline{1}]_{\beta} 1.49^{\circ}$ from $[01 \overline{1}]_{\alpha_{1}^{\prime \prime}}$ & {$[11 \overline{1}]_{\beta} 2.61^{\circ}$ from $[110]_{\alpha_{2}^{\prime \prime}}$} \\
\hline$[1 \overline{1} 1]_{\beta} 1.49^{\circ}$ from $[101]_{\alpha_{1}^{\prime \prime}}$ & {$[1 \overline{1} 1]_{\beta} 2.63^{\circ}$ from $[0 \overline{1} 1]_{\alpha_{2}^{\prime \prime}}$} \\
\hline$[1 \overline{1} \overline{1}]_{\beta} 0.73^{\circ}$ from $[10 \overline{1}]_{\alpha_{1}^{\prime \prime}}$ & {$[1 \overline{1} \overline{1}]_{\beta} 2.74^{\circ}$ from $[1 \overline{1} 0]_{\alpha}$} \\
\hline$[100]_{\beta} 0.86^{\circ}$ from $[110]_{\alpha_{1}^{\prime \prime}}$ & {$[100]_{\beta} 0.22^{\circ}$ from $[101]_{\alpha_{2}^{\prime}}$} \\
\hline$[0 \overline{1} 0]_{\beta} 1.61^{\circ}$ from $[1 \overline{1} 0]_{\alpha_{1}^{\prime \prime}}$ & {$[00 \overline{1}]_{\beta} 3.20^{\circ}$ from $[1$} \\
\hline
\end{tabular}

Table 3

Orientation relationships obtained using lattice parameters measured in by Talling et al. [3].

\begin{tabular}{l}
$\beta$ and $\alpha^{\prime \prime}$ twin $1 \quad \beta$ and $\alpha^{\prime \prime}$ twin 2 \\
\hline$(1 \overline{1} 0)_{\beta} 2.96^{\circ}$ from $(100)_{\alpha_{1}^{\prime \prime}}(101)_{\beta} 1.09^{\circ}$ from $(100)_{\alpha_{2}^{\prime \prime}}$ \\
$(10 \overline{1})_{\beta} 2.39^{\circ}$ from $(010)_{\alpha_{1}^{\prime \prime}}(010)_{\beta} 1.11^{\circ}$ from $(010)_{\alpha_{2}^{\prime \prime}}$ \\
$(001)_{\beta} 3.02^{\circ}$ from $(001)_{\alpha_{1}^{\prime \prime}}(101)_{\beta} 0.34^{\circ}$ from $(001)_{\alpha_{2}^{\prime \prime}}$ \\
\hline$[111]_{\beta} 1.74^{\circ}$ from $[011]_{\alpha_{1}^{\prime \prime}}[111]_{\beta} 0.81^{\circ}$ from $[011]_{\alpha_{2}^{\prime \prime}}$ \\
{$[11 \overline{1}]_{\beta} 3.26^{\circ}$ from $[01 \overline{1}]_{\alpha_{1}^{\prime \prime}}[11 \overline{1}]_{\beta} 1.07^{\circ}$ from $[110]_{\alpha_{2}^{\prime \prime}}$} \\
{$[1 \overline{1} 1]_{\beta} 2.49^{\circ}$ from $[101]_{\alpha_{1}^{\prime \prime}}[1 \overline{1} 1]_{\beta} 0.58^{\circ}$ from $[0 \overline{1} 1]_{\alpha_{2}^{\prime \prime}}$} \\
{$[1 \overline{1} \overline{1}]_{\beta} 3.40^{\circ}$ from $[10 \overline{1}]_{\alpha_{1}^{\prime \prime}}[1 \overline{1} \overline{1}]_{\beta} 1.12^{\circ}$ from $[1 \overline{1} 0]_{\alpha_{2}^{\prime \prime}}$} \\
{$[100]_{\beta} 1.72^{\circ}$ from $[110]_{\alpha_{1}^{\prime \prime}}[100]_{\beta} 0.98^{\circ}$ from $[101]_{\alpha_{2}^{\prime \prime}}$} \\
{$[0 \overline{1} 0]_{\beta} 3.40^{\circ}$ from $[1 \overline{1} 0]_{\alpha_{1}^{\prime \prime}}[00 \overline{1}]_{\beta} 0.57^{\circ}$ from $[10 \overline{1}]_{\alpha_{2}^{\prime \prime}}$}
\end{tabular}

